\title{
User Curiosity Factor in Determining Serendipity of Recommender System
}

\author{
Arseto Satriyo Nugroho ${ }^{1}$, Igi Ardiyanto ${ }^{2}$, Teguh Bharata Adji ${ }^{3}$
}

\begin{abstract}
Recommender rystem (RS) is created to solve the problem by recommending some items among a huge selection of items that will be useful for the e-commerce users. RS prevents the users from being flooded by information that is irrelevant for them. Unlike information retrieval (IR) systems, the RS system's goal is to present information to the users that is accurate and preferably useful to them. Too much focus on accuracy in RS may lead to an overspecialization problem, which will decrease its effectiveness. Therefore, the trend in RS research is focusing beyond accuracy methods, such as serendipity. Serendipity can be described as an unexpected discovery that is useful. Since the concept of a recommendation system is still evolving today, formalizing the definition of serendipity in a recommendation system is very challenging. One known subjective factor of serendipity is curiosity. While some researchers already addressed curiosity factor, it is found that the relationships between various serendipity component as perceived by the users and their curiosity levels is still yet to be researched. In this paper, the method to determine user curiosity model by considering the variation of rated items was presented, then relation to serendipity components using existing user feedback data was validated. The finding showed that the curiosity model was related to some userperceived values of serendipity, but not all. Moreover, it also had positive effect on broadening the user preference.
\end{abstract}

Keywords-Recommender System, Serendipity, Relevance, Novelty, Unexpectedness, Evaluation Metrics, Curiosity.

\section{INTRODUCTION}

\section{A. A Need for Useful Recommender System}

Along with the rapid development of information technology, our life is increasingly inseparable from digitalbased platforms. They start from e-commerce-based online businesses to digital multimedia entertainment that can be easily accessed using the internet. The choice of goods and services is increasingly diverse and easily accessible. Many options which are available on the one hand can be helpful, but on the other hand it can also make it difficult for consumers to choose which product fits their needs [1].

From the producer and service provider point of view, the abundance of product variations coupled with the ease of access to information and distribution will shift the marketing focus from a few popular products (hits market) to many less popular products (niche market). This concept is called the long tail concept [2]. Furthermore, the concept can shift the Pareto

1,2,3 Department of Electrical and Information Engineering, Faculty of Engineering, Universitas Gadjah Mada, Jln. Grafika No. 2, Kampus UGM, Yogyakarta, 55281, INDONESIA (phone: 0274552305; email: 1arseto.s.n@mail.ugm.ac.id, 2igi@ugm.ac.id, 3adji@ugm.ac.id) principle commonly practiced in marketing nowadays, as in Fig. 1 [3].

A recommender system (RS) primary function is to provide item recommendations for users [1], [4], where the item is a piece of information that refers to a physical object or digital products, services, or processes suggested by the recommendation system to users through the interaction via web, email, or text message [5]. RS is often found in various domains such as e-commerce, entertainment, services, social networking, and other digital content [1]. This recommendation system must pay attention to the users' preferences so that user recommendations are helpful for them [1]. From the seller and manufacturer's perspective, a recommendation system can potentially help them recommending less popular items within the long tail [3].

The success of RS is measured not only by how accurate it is in recommending items based on user preferences, but also by how the results of these recommendations can be helpful to users [4]. RS that only pay attention to accuracy tend to experience overspecialization problems, which lead the system to provide recommendations that are too similar to items that have been seen or consumed before [6], [7]. It will narrow the users' point of view [8], reducing user satisfaction with the system [5]. It is caused by only considering the accuracy factor and ignoring the benefits of recommendation [7]. Therefore, it is essential to pay attention to other factors besides accuracy that influence [9] the quality of the recommendations [10]-[12]. One of these factors is serendipity, which can be interpreted as a surprising and unexpected discovery of an item that may not be encountered or very difficult to be encountered in other circumstances [1].

\section{B. Serendipity in Recommender System}

Serendipity itself is a difficult word to translate, which in general can be interpreted as an unexpected but profitable discovery of something new [13]. The concept of serendipity is also closely related to subjectivity. Thus, serendipity becomes challenging to learn and implement [14], [15]. This subjective nature makes the prediction of serendipity different for each individual. Some studies argue that the nature of a user's curiosity affects the serendipitous definition of the user, which in turn also affects user satisfaction [16], [17].

The subjectivity of serendipity depends mainly on two factors, namely user knowledge and user curiosity [18]. The psychological theory defines two assessments of curiosity, namely 1) an assessment of something new, unexpected, and complex, and 2) an assessment of a person's ability to understand something new and complex [19], which are then defined as novelty check and coping potential check [18]. The first score is calculated based on the difference between the 


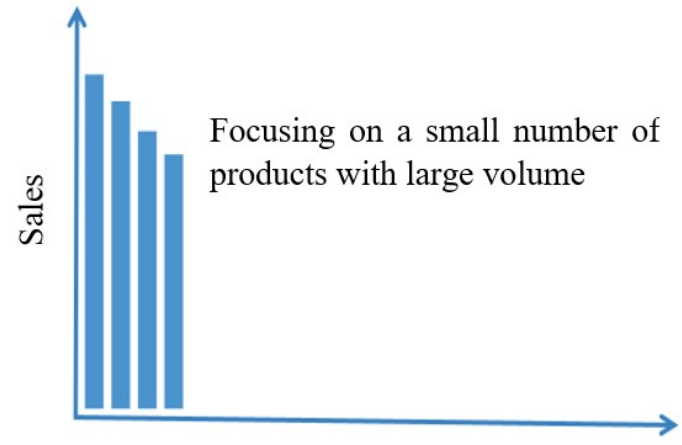

Number of products

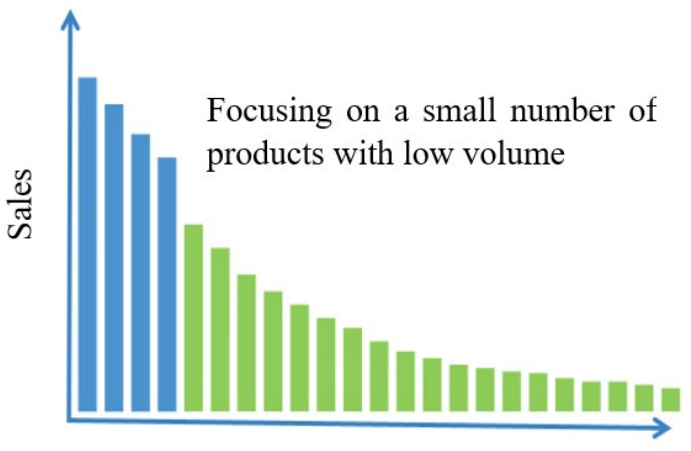

Number of products

Fig. 1 Hits market vs niche market.

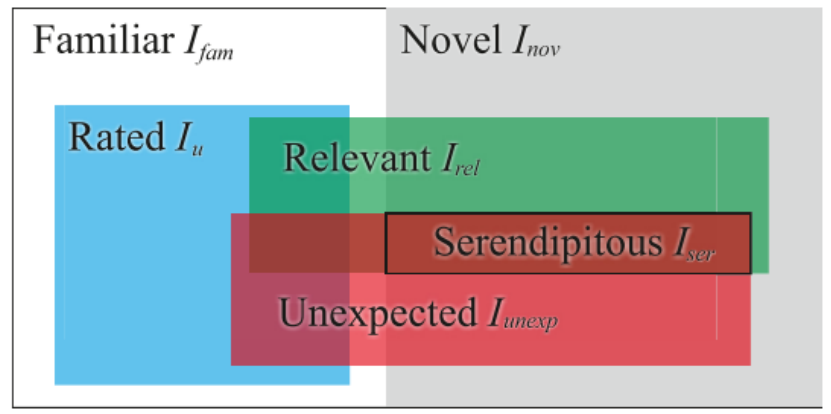

Fig. 2 Euler diagram of an item from the users' perspective.

item and the user profile. The second score is calculated based on the diversity of items in the user's profile. The conclusion is that using the user curiosity parameter in making recommendations can balance the serendipity and accuracy. It is also found that users who have a more comprehensive genre profile were more open to serendipitous recommendations.

\section{Goal and Contribution}

This study aims to find the relationship among the serendipity metric components, vis popularity, and similarity to user profiles. The perception of serendipity is from the users' point of view based on the survey results regarding user curiosity as a psychological factor. Previous research [18] divided user groups by two, namely having five or more genres in their profile and having less than five genres in their profile. The proposed method was to measure curiosity degree by counting distinct genres rated by the user. In this paper, user curiosity measurement based on rating genre diversity was proposed.

\section{SERENDIPITY}

Serendipity can be measured by comparing the output of a recommendation system with a primitive recommendation system based on accuracy [20]. This approach is called full metric because it measures serendipity as a whole. The advantage of this approach is serendipity value can be known comprehensively. Another approach is using the component metric. The component metric measures serendipity components, including relevance, novelty, and unexpectedness. Measurement using this method has the advantage if several aspects related to serendipity need to be described [21]. Fig. 2 describes the profile of an item regarding serendipity metrics [21].

Apart from the advantages which have been mentioned, both approaches still have disadvantages [5]. The complete metric method has a particular weakness, which is too sensitive to the primitive recommendation system when it is used for comparison [5], [22], [23]. If the recommendation system parameters used for comparison are changed, it will significantly affect the results of the serendipity measurement on the results of the recommendation system being tested. Meanwhile, the component method can be wrong in estimating the serendipity value because what is assessed is the metric of each component [21].

A novelty component in serendipity can be represented in two approaches. The first approach uses the item popularity and the second approach uses the similarity with the user profile. The novelty is defined as the opposite of popularity [24], assuming that the popular item is generally known to users through other media. Therefore, the popularity of an item is an essential component in serendipity [21]. The similarity of an item with the user profile is also necessary to consider because users may tend to search for the one according to their preferences and which they are probably familiar with [24]. While the literature review shows that the serendipitous item is less popular and relevant to the user profile, thus the conclusion cannot be drawn yet [25].

Recommending item that is unpopular, not similar to a user profile, but still relevant at the same time is still challenging to provide valuable results for users, at least for two reasons. First, a non-popular item is likely to be of low quality, so that users will judge it as an irrelevant item. Second, if there is too much 
difference between the item recommendation and the users' profile, the users may dislike it [21].

Along with novelty, diversity is identified as a key dimension in the utility of recommendations in a real scenario [24]. The diversity within a collection item is related to how different it is between one item and another.

The notion of diversity in RS stems comes from a concept in IR research [26]. In information retrieval (IR) literature, the document obtained valuation is influenced not only by the similarity with the user query, but also by the similarity between the documents obtained [27]. Recent research [28] found that accuracy, serendipity, and diversity in RS were interrelated. Higher diversity could worsen accuracy and worsen or improve serendipity depending on how much it was added. Some known metrics for measuring serendipity are explained in the following section.

\section{A. Relevance}

Relevant items are items that are liked, consumed, or attract the users' attention, depending on the recommendation system scenario [21]. Recommended items also must be useful and affect users' emotions positively [29], [30].

Relevance in the recommendation context is user-specific interest for items. Depending on the available observations, relevance can be modeled accordingly [24]. In the case of user rating observation, the probability of the user to like an item can be modeled by a heuristic mapping between rating value and relevance probability. For instance, the expected reciprocal rank (ERR) metric shown in (1) can be used [31], where $g$ is a utility function derived from ratings by user $u$ for the movie $i$. In addition, $g_{\max }$ is the maximum value of $g(u, i)$ for each specific user.

$$
p(r e l \mid i, u) \sim \frac{2^{g(u, i)}-1}{2^{g \max }} .
$$

In Movielens, different algorithms for predicting user rating can be chosen by the user [25]. These algorithms outputs predictedRating as a feature unique to a combination of user and movies.

\section{B. Novelty and Popularity}

Novelty can be measured using different approaches. The most relevant approaches are using item popularity and item distance respectively [24]. In this paper, a popularity-based approach was used to measure the novelty of an item relative to a user.

Item discovery is formulated as the probability that an item is not yet observed earlier [24], as shown in (2).

$$
\operatorname{pop}(i \mid \theta)=p(\operatorname{seen} \mid i, \theta) .
$$

Contextual variable $\theta$ represents any element on which item discovery may depend. It might be a specific user, group of users, time interval, item source, etc. Considering the probability of item discovery, the novelty of an item can be defined as the inverse of its popularity as shown in (3) [24].

$$
\operatorname{nov}(i \mid \theta)=1-p(\operatorname{seen} \mid i, \theta) \text {. }
$$

To emphasize highly novel items, the log of the inverse popularity is considered as shown in (4) [24].

$$
\operatorname{nov}(i \mid \theta)=-\log _{2} p(\operatorname{seen} \mid i, \theta) \text {. }
$$

Alternatively, from (2), less popular items can also be emphasized by applying log function [25] as shown in (5).

$$
\log \operatorname{pop}(i \mid \theta)=\log (p(\operatorname{seen} \mid i, \theta)) .
$$

In movie recommendation context, $p(\operatorname{seen} \mid i, \theta)$ can be represented by whether the user already rated it or not.

\section{Unexpectedness}

Unexpectedness can be measured using two different approaches, the first approach is by comparing results from a novel recommendation system with a traditional, accuracybased recommendation system [20]. As shown in (6), with $s_{i}(i=1 \cdots N)$ represents item $i$ from the recommendation list, $\operatorname{Pr}\left(s_{i}\right)$ denotes the level of belief generated by the recommendation system. $\operatorname{Prim}\left(s_{i}\right)$ denotes the level of belief generated. And lastly, isrel $\left(s_{i}\right)$ denotes relevance with the user profile.

$$
\text { unexp }=\frac{1}{N} \sum_{i=1}^{N} \max \left(\operatorname{Pr}\left(s_{i}\right)-\operatorname{Prim}\left(s_{i}\right), 0\right) \cdot i \operatorname{srel}\left(s_{i}\right) .
$$

The second approach is by calculating unexpectedness as a component calculated from the recommendation results. One method is by calculating the distance of recommended items to the users' expected item set [30]. The expected set by user $E_{u}$ will have an unexpectedness value of 0 . The farther the distance of an item from the expected set, which is represented by $\delta_{u, i}$, the higher the unexpectedness value as shown in (7), to the point that the item is not relevant to the users anymore.

$$
\delta_{u, i}=\operatorname{dist}\left(i ; E_{u}\right)
$$

where $\operatorname{dist}\left(i ; E_{u}\right)$ is the distance of item $i$ from $E_{u}$ for user $u$. It is needed to somewhat limit the distance to prevent it from becoming irrelevant. Then, the unexpectedness of item $i$ concerning $E_{u}$ is defined as some unimodal function of this distance defined in (8).

$$
\Delta\left(\delta_{u, i} ; \delta_{u}^{*}\right)
$$

where $\delta_{u}^{*}$ is the best, most preferred, unexpected distance from the expected set $E_{u}$ for user $u$.

\section{Diversity}

Diversity can be measured as the average pairwise distance between items in the list as shown in (9) [32].

$$
\operatorname{Diversity}(R)=\frac{\sum_{i \in R} \sum_{j \in R \backslash\{i\}} \operatorname{dist}(i, j)}{|R|(|R|-1)} .
$$

Conversely, by measuring similarity, the value of diversity is the opposite of that diversity. The similarity value can be obtained from the aggregate of pairwise similarity [33]. This method is further developed using different parameters for the distance function [26]. For distance calculations based on the content descriptor, distance functions can use taxonomy-based metrics [33], the complement of Jaccard similarity [24], or the 
TABLE I

SERENDIPITY COMPONENTS RATED BY USERS

\begin{tabular}{|c|l|l|}
\hline Symbol & Component Name & \multicolumn{1}{|c|}{ Detail } \\
\hline $\mathrm{S}_{1}$ & Strict Novelty & $\begin{array}{l}\text { The user has never heard about } \\
\text { the movie }\end{array}$ \\
\hline $\mathrm{S}_{2}$ & $\begin{array}{l}\text { Motivational } \\
\text { Novelty }\end{array}$ & $\begin{array}{l}\text { The user already knew the } \\
\text { movie but has not seen it yet }\end{array}$ \\
\hline $\mathrm{S}_{3}$ & $\begin{array}{l}\text { Unexpected } \\
\text { Relevance }\end{array}$ & $\begin{array}{l}\text { The user does not expect the } \\
\text { item to be relevant }\end{array}$ \\
\hline $\mathrm{S}_{4}$ & Unexpected Find & $\begin{array}{l}\text { The user does not expect to find } \\
\text { the movie without using the } \\
\text { recommender system }\end{array}$ \\
\hline $\mathrm{S}_{5}$ & $\begin{array}{l}\text { Unexpected } \\
\text { Implicit }\end{array}$ & $\begin{array}{l}\text { The movie is very different } \\
\text { from the movies that the user } \\
\text { usually consumes }\end{array}$ \\
\hline $\mathrm{S}_{6}$ & $\begin{array}{l}\text { Unexpected } \\
\text { Recommendation }\end{array}$ & $\begin{array}{l}\text { The user does not expect to be } \\
\text { recommended with this movie }\end{array}$ \\
\hline $\mathrm{S}_{7}$ & User Satisfaction & $\begin{array}{l}\text { The user is satisfied by the } \\
\text { recommendation }\end{array}$ \\
\hline $\mathrm{S}_{8}$ & $\begin{array}{l}\text { Preference } \\
\text { Broadening }\end{array}$ & $\begin{array}{l}\text { The recommendation broadens } \\
\text { the user preference }\end{array}$ \\
\hline & &
\end{tabular}

TABLE II

GENRE DATA AS REPRESENTED IN DATASET

\begin{tabular}{|c|l|}
\hline movieId & \multicolumn{1}{|c|}{ Genres } \\
\hline 1 & Adventure,Animation,Children,Comedy,Fantasy \\
\hline 2 & Adventure,Children,Fantasy \\
\hline 3 & Comedy,Romance \\
\hline 4 & Comedy,Drama,Romance \\
\hline 5 & Comedy \\
\hline 6 & Action,Crime,Thriller \\
\hline 7 & Comedy,Romance \\
\hline 8 & Adventure,Children \\
\hline 9 & Action \\
\hline 10 & Action,Adventure,Thriller \\
\hline 11 & Comedy,Drama,Romance \\
\hline
\end{tabular}

TABLE III

MOVIE GENRE PROFILE

\begin{tabular}{|c|c|c|c|c|c|}
\hline movieId & Adventure & Animation & Children & Comedy & $\ldots$ \\
\hline 1 & 1 & 1 & 1 & 1 & $\ldots$ \\
\hline 2 & 1 & 0 & 1 & 0 & $\ldots$ \\
\hline 3 & 0 & 0 & 0 & 1 & $\ldots$ \\
\hline 4 & 0 & 0 & 0 & 1 & $\ldots$ \\
\hline 5 & 0 & 0 & 0 & 1 & $\ldots$ \\
\hline 6 & 0 & 0 & 0 & 0 & $\ldots$ \\
\hline 7 & 0 & 0 & 0 & 1 & $\ldots$ \\
\hline 8 & 1 & 0 & 1 & 0 & $\ldots$ \\
\hline 9 & 0 & 0 & 0 & 0 & $\ldots$ \\
\hline 10 & 1 & 0 & 0 & 0 & $\ldots$ \\
\hline 11 & 0 & 0 & 0 & 1 & $\ldots$ \\
\hline$\ldots$ & $\ldots$ & $\ldots$ & $\ldots$ & $\ldots$ & $\ldots$ \\
\hline
\end{tabular}

complement of cosine similarity of term vectors [34]. For distance calculations based on the rating vector, the method that can be used is the Hamming distance [35], the complement of Pearson correlation [24], or the complement of cosine similarity [36].

\section{EXPERIMENTAL SETUP}

\section{A. The Dataset}

This experiment used survey results based on the Movielens dataset where users were asked how serendipitous particular movies were to them [25]. The dataset used was rating data in training.csv, survey data in answers.csv, and movie data in movies.csv. The data was processed with $\mathrm{R}$ using RStudio. This dataset provides this research's ground truth regarding user perception of various components of serendipity detailed in Table I [25].

\section{B. Data Preparation and Pre-processing}

Each movie in movies.csv has multiple genres associated with it, written as comma-separated-values as shown in Table II. Each movie was then profiled by its genres, resulting in a table with genres as columns and a value of 0 or 1 . Each value represents the genre associated with the movie as shown in Table III, this will be this research's movie genre profile.

To get items which already rated by the user, training.csv file was used. For validating the results, answers.csv was used to compare the calculated metrics with real user feedback. Data from training.csv dataset with timestamps older than the oldest timestamp in the answers dataset were excluded. After that, the movie genre profile and the user rating data in training.csv were merged and summarized by userId, resulting in Table IV.

\section{Calculating User's Curiosity Profile}

Whether it is a good or bad rating, any movie rated by a user signifies the user's potential to like a wider range of genres. Considering $r\left(g, u_{i}\right)$ as the number of movies rated by user $i$ with genre $g$, the user's curiosity profile $C\left(u_{i}\right)$ is created by counting the number of unique genres rated by the user, as formulated in (10).

$$
C\left(u_{i}\right)=\sum_{i}^{n}\left[r\left(g, u_{i}\right)>0\right] .
$$

By applying this formula to the user genre profile in Table IV, curiosity profile was created as in Table V.

\section{Calculating Movie Popularity Metric}

In their literature review, [21] stated that a less popular item is more likely to be serendipitous. Hence, popularity calculation is included where $r_{m i}$ is the number of ratings associated with the movie in the training dataset [25]. The formulation is in (11).

$$
\operatorname{logpop}\left(m_{i}\right)=\ln \left(r_{m i}\right) .
$$

\section{E. Relevance to User Profile}

In the same review, [21] also stated that relevant items also more likely to be serendipitous. Relevance to the user profile is predicted by the MovieLens system and provided in the predictedRating field in the answers dataset [25]. Assuming the higher predictedRating represents higher relevance to the user profile, the relevance to the user profile can be stated as (12).

$$
\operatorname{Rel}\left(m_{i}, u_{j}\right)=\operatorname{predictedRating}\left(m_{i}, u_{j}\right) \text {. }
$$


TABLE IV

USER GENRE PROFILE

\begin{tabular}{|c|c|c|c|c|c|}
\hline userId & $\begin{array}{c}\text { Sum } \\
\text { Adventure }\end{array}$ & $\begin{array}{c}\text { Sum } \\
\text { Animation }\end{array}$ & $\begin{array}{c}\text { Sum } \\
\text { Children }\end{array}$ & $\begin{array}{c}\text { Sum } \\
\text { Comedy }\end{array}$ & $\ldots$ \\
\hline 100032 & 0 & 0 & 0 & 0 & $\ldots$ \\
\hline 100053 & 7 & 0 & 1 & 17 & $\ldots$ \\
\hline 100058 & 1 & 1 & 0 & 5 & $\ldots$ \\
\hline 100067 & 0 & 0 & 0 & 0 & $\ldots$ \\
\hline 100076 & 1 & 1 & 2 & 6 & $\ldots$ \\
\hline 100093 & 4 & 0 & 2 & 8 & $\ldots$ \\
\hline 100094 & 1 & 0 & 0 & 4 & $\ldots$ \\
\hline 100095 & 0 & 0 & 2 & 0 & $\ldots$ \\
\hline 100101 & 0 & 8 & 0 & 5 & $\ldots$ \\
\hline 100103 & 3 & 1 & 3 & 1 & $\ldots$ \\
\hline$\ldots$ & $\ldots$ & $\ldots$ & $\ldots$ & $\ldots$ & $\ldots$ \\
\hline
\end{tabular}

TABLE V

USER CURIOSITY PROFILE

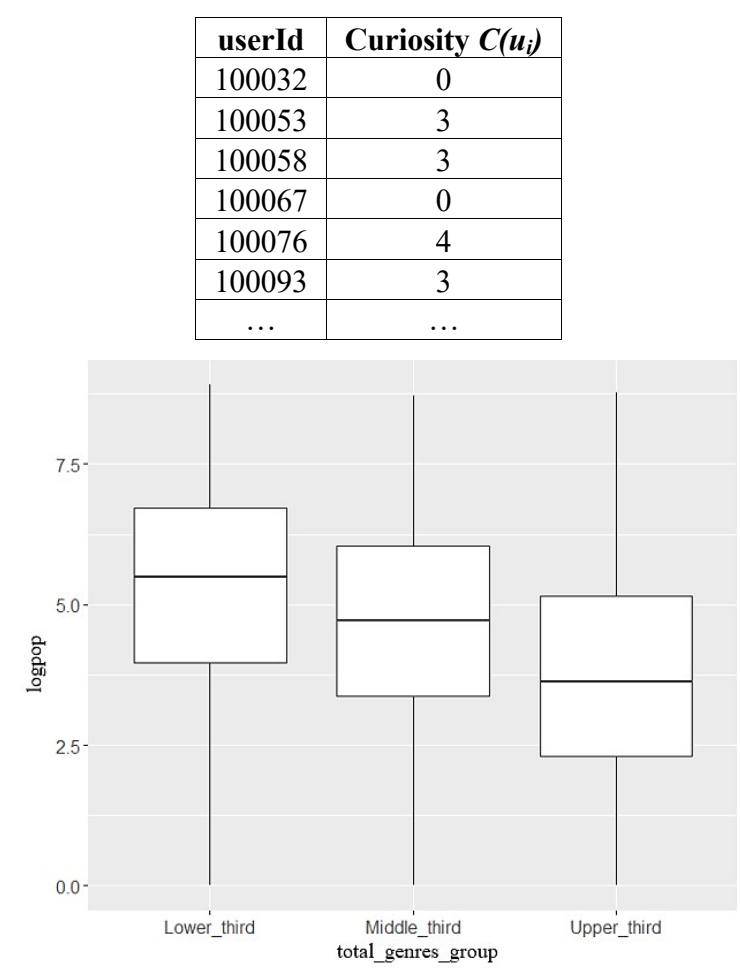

Fig. 3 Relationship between user genre diversity with the popularity of the popularity and relevance effect on serendipity components.

\section{F. Predicting Component Relationships Using Regresion Models}

Since ordinal categorical datas are used as dependent variables, a cumulative link regression model (CLM) [37] is used. The regression models predicted the relationships between user curiosity, movie popularity, and relevance to a user profile with user's perception regarding serendipity components, as shown in Table I.

Curiosity, popularity, and relevance are used as the predictors or the independent variables, with various serendipity definitions as responses or dependent variables. The first two models, (13) and (14), are based on the literature review in [21] and further experimented in [25], which conclude that the popularity of an item and its relevance to the user affect serendipity. The third model is based on the proposed curiosity model stated in (15).

$$
\begin{gathered}
s_{\text {comp }} \sim \alpha+a_{1} * \operatorname{logpop}\left(r_{m i}\right) \\
s_{\text {comp }} \sim \alpha+a_{2} * \text { predictedRating }\left(m_{i}, u_{j}\right) \\
s_{\text {comp }} \sim \alpha+a_{3} * C\left(u_{i}\right) .
\end{gathered}
$$

$S_{\text {comp }}$ is varied between six definitions of serendipity components and two additional metrics shown in Table I. Variable $\alpha$ is the regression intercept, while $a_{1}, a_{2}$, and $a_{3}$ are the respective regression coefficients for each independent variable.

\section{RESUlT AND DisCUSSION}

\section{A Curiosity Relationship with Other Metrics}

Our curiosity model is defined by counting unique genres rated by the user, which produces an integer value between 1 and 19, as there are 19 unique genres present in the dataset. For easier visualization, the value range was grouped into three ranges: lower, middle, and upper, based on the quantile range of the unique genre per-user data. Plotting it against the popularity of the movie (logpop), users having more diverse genre ratings seemed to rate more unpopular movies as depicted in Fig. 3.

It does not always mean that users with the more diverse genre only rates less popular items, but it shows that less popular movie also tends to have fewer common genres. Therefore, users having a more diverse genre means that they have more ratings in less popular movies.

In the previous article, it is assumed that serendipitous items are items that less popular but are relevant to the user profile [21]. Regression models mentioned in (13) and (14) were used to check whether this a ssumption applies to this dataset. The regression result is shown in Table VI.

Using a statistical significance threshold of 0.01 , it is concluded that movie popularity negatively affects serendipity. It can be seen from the negative values of the significant estimates. In addition, some serendipity definitions, namely unexpected relevance and motivational novelty, do not relate to item popularity. Moreover, item popularity does not significantly affect user satisfaction but it does negatively affect user preference broadening. It is to be expected because the more popular the movie is recommended to the users, the more likely the users already knows about it.

Predicted rating variables have a more positive effect on serendipity definitions than the previous research [25]. It was hinted at by mostly positive and significant coefficients. Its impact on user satisfaction and preference broadening was also positive and statistically significant.

\section{G. User Curiosity Effects on Serendipity Components}

The proposed regression model added a user curiosity metric based on genre diversity in the user profile, resulting in 
TABLE VI

REGRESSION ESTIMATES FOR LOGPOP AND PREDICTED RATING

\begin{tabular}{|l|c|c|c|c|c|}
\hline \multicolumn{6}{|c|}{ Logpop } \\
\hline & Estimate & Std Error & $\boldsymbol{z}$ value & $\operatorname{Pr}(>|z|)$ & Significance \\
\hline $\mathrm{S}_{1}$ & -0.07417 & 0.02792 & -2.657 & 0.00788 & 0.001 \\
\hline $\mathrm{S}_{2}$ & 0.003803 & 0.025345 & 0.15 & 0.881 & \\
\hline $\mathrm{S}_{3}$ & 0.04067 & 0.02588 & 1.571 & 0.116 & \\
\hline $\mathrm{S}_{4}$ & -0.09718 & 0.02472 & -3.931 & $8.48 \mathrm{E}-05$ & 0 \\
\hline $\mathrm{S}_{5}$ & -0.10899 & 0.02489 & -4.378 & $1.20 \mathrm{E}-05$ & 0 \\
\hline $\mathrm{S}_{6}$ & -0.09629 & 0.02509 & -3.838 & 0.000124 & 0 \\
\hline $\mathrm{S}_{7}$ & 0.053 & 0.02653 & 1.998 & 0.0458 & 0.01 \\
\hline $\mathrm{S}_{8}$ & -0.09207 & 0.02477 & -3.718 & 0.000201 & 0 \\
\hline & \multicolumn{5}{|c|}{ Predicted Rating } \\
\hline & Estimate & Std Error & $\boldsymbol{z}$ value & $\operatorname{Pr}(>|z|)$ & Significance \\
\hline $\mathrm{S}_{1}$ & 0.2475 & 0.1098 & 2.254 & 0.0242 & 0.01 \\
\hline $\mathrm{S}_{2}$ & 0.3594 & 0.1017 & 3.532 & 0.000412 & 0 \\
\hline $\mathrm{S}_{3}$ & 0.4656 & 0.1039 & 4.481 & $7.43 \mathrm{E}-06$ & 0 \\
\hline $\mathrm{S}_{4}$ & 0.14361 & 0.09652 & 1.488 & 0.137 & \\
\hline $\mathrm{S}_{5}$ & 0.09074 & 0.09574 & 0.948 & 0.343 & \\
\hline $\mathrm{S}_{6}$ & -0.23322 & 0.09929 & -2.349 & 0.0188 & 0.01 \\
\hline $\mathrm{S}_{7}$ & 0.5295 & 0.1055 & 5.019 & $5.19 \mathrm{E}-07$ & 0 \\
\hline $\mathrm{S}_{8}$ & 0.34973 & 0.09755 & 3.585 & 0.000337 & 0 \\
\hline & & & & \\
\hline
\end{tabular}

TABLE VII

REGRESSION ESTIMATES FOR TOTAL GENRES CURIOSITY

\begin{tabular}{|l|c|c|c|c|c|}
\hline & Estimate & Std Error & $\boldsymbol{z}$ value & $\boldsymbol{P r}(>|\boldsymbol{z}|)$ & Significance \\
\hline $\mathrm{S}_{1}$ & 0.008746 & 0.011718 & 0.746 & 0.455 & \\
\hline $\mathrm{S}_{2}$ & 0.001583 & 0.010497 & 0.151 & 0.88 & \\
\hline $\mathrm{S}_{3}$ & -0.008473 & 0.010889 & -0.778 & 0.436 & \\
\hline $\mathrm{S}_{4}$ & 0.01947 & 0.01029 & 1.893 & 0.0584 & 0.1 \\
\hline $\mathrm{S}_{5}$ & 0.03358 & 0.01042 & 3.221 & 0.00128 & 0.001 \\
\hline $\mathrm{S}_{6}$ & 0.03551 & 0.0105 & 3.382 & 0.000718 & 0 \\
\hline $\mathrm{S}_{7}$ & -0.009097 & 0.011086 & -0.821 & 0.412 & \\
\hline $\mathrm{S}_{8}$ & 0.03707 & 0.01038 & 3.571 & 0.000356 & 0 \\
\hline
\end{tabular}

coefficients presented in Table VII. Using a statistical significance threshold of 0.01 , it can be concluded that user genre diversity only affects two serendipity definitions, namely unexpected implicit $\left(\mathrm{S}_{5}\right)$ and unexpected recommendation $\left(\mathrm{S}_{6}\right)$. Additionally, it has better performance in predicting user preference broadening $\left(\mathrm{S}_{8}\right)$.

\section{CONCLUSION}

The experiment has shown that some serendipity definition according to the users' response corresponds to the popularity of the movie and its relevance to the user profile. Especially the movie relevance, represented as predicted rating value, significantly affects item popularity in predicting the serendipitous results. The result shows that the user curiosity factor affects some users' perception of serendipitous items and positively affects preference broadening but it does not relate to user satisfaction.

While the user curiosity factor proved to affect some serendipity components, the significance can still be improved. The limitation in this study is by using existing offline data from another experiment. For further study, it is suggested to use an online method to assess user feedback regarding the curiosity factor and its effect on serendipity perception.

\section{REFERENCES}

[1] F. Ricci, B. Shapira, and L. Rokach, Eds., Recommender Systems Handbook, 2nd ed., New York, USA: Springer US, 2015.

[2] C. Anderson, "The Long Tail: Why the Future of Business Is Selling Less of More," New York, USA: Hachette Books, 2006.

[3] H. Yin, B. Cui, J. Li, J. Yao, and C. Chen, "Challenging the Long Tail Recommendation," Proceedings of the VLDB Endowment (PVLDB), 2012, pp. 896-907.

[4] R. Burke, "Hybrid Web Recommender Systems," in The Adaptive Web: Methods and Strategies of Web Personalization, P. Brusilovsky, A. Kobsa, and W. Nejdl, Eds., Berlin, Germany: Springer-Verlag, 2007, pp. 377-408.

[5] D. Kotkov, J. Veijalainen, and S. Wang, "Challenges of Serendipity in Recommender Systems," Proceedings of the 12th International Conference on Web Information Systems and Technologies, Vol. 2, 2016, pp. 251-256.

[6] S.M. McNee, J. Riedl, and J. Konstan, "Being Accurate Is not Enough: How Accuracy Metrics Have Hurt Recommender Systems," CHI '06 Extended Abstracts on Human Factors in Computing Systems, 2006, pp. 1097-1101.

[7] M. de Gemmis, P. Lops, G. Semeraro, and C. Musto, "An Investigation on the Serendipity Problem in Recommender Systems," Information Processing \& Management, Vol. 51, No. 5, pp. 695-717, Sep. 2015.

[8] E. Pariser, The Filter Bubble: What The Internet Is Hiding from You. London, England: Penguin Group, 2011.

[9] J.L. Herlocker, J.A. Konstan, L.G. Terveen, and J.T. Riedl, "Evaluating Collaborative Filtering Recommender Systems," ACM Transactions on Information Systems, Vol. 22, No. 1, pp. 5-53, Jan. 2004.

[10] P. Castells, J. Wang, R. Lara, and D. Zhang, "Workshop on Novelty and Diversity in Recommender Systems-DiveRS 2011," Proceedings of the 5th ACM Conference on Recommender Systems, 2011, pp. 393-394.

[11] N. Hurley and M. Zhang, "Novelty and Diversity in Top-N Recommendation-Analysis and Evaluation," ACM Transactions on Internet Technology, Vol. 10, No. 4, pp. 1-30, Mar. 2011.

[12] Y.C. Zhang, D.Ó. Séaghdha, D. Quercia, and T. Jambor, "Auralist: Introducing Serendipity into Music Recommendation," Proceedings of the 5th ACM International Conference on Web Search and Data Mining, 2012, pp. 13-22.

[13] D. Martin. (2008) Most Untranslatable Word. [Online], https:/www.todaytranslations.com/news/most-untranslatable-word, access date: Nov. 23, 2018.

[14] L. Iaquinta, M. de Gemmis, P. Lops, G. Semeraro, M. Filannino, and P. Molino, "Introducing Serendipity in a Content-Based Recommender System," Proceedings of the 8th International Conference on Hybrid Intelligent Systems, 2008, pp. 168-173.

[15] A. Foster and N. Ford, "Serendipity and Information Seeking: An Empirical Study," Journal of Documentation, Vol. 59, No. 3, pp. 321340, Jan. 2003.

[16] P. Zhao and D.L. Lee, "How Much Novelty Is Relevant? It Depends on Your Curiosity," Proceedings of the 39th International ACM SIGIR Conference on Research and Development in Information Retrieval, 2016, pp. 315-324.

[17] L. Chen, Y. Yang, N. Wang, K. Yang, and Q. Yuan, "How Serendipity Improves User Satisfaction with Recommendations? A Large-Scale User Evaluation," Proceedings of the 2019 World Wide Web Conference, 2019, pp. $240-250$. 
[18] V. Maccatrozzo, M. Terstall, L. Aroyo, and G. Schreiber, "SIRUP: Serendipity in Recommendations via User Perceptions," Proceedings of the 22nd International Conference on Intelligent User Interfaces, 2017, pp. 35-44.

[19] P.J. Silvia, "Emotional Responses to Art: From Collation and Arousal to Cognition and Emotion," Review of General Psychology, Vol. 9, No. 4, pp. 342-357, Dec. 2005.

[20] T. Murakami, K. Mori, and R. Orihara, "Metrics for Evaluating the Serendipity of Recommendation Lists," Proceedings of the 2007 Conference on New Frontiers in Artificial Intelligence, 2007, pp. 40-46.

[21] D. Kotkov, S. Wang, and J. Veijalainen, "A Survey of Serendipity in Recommender Systems," Knowledge-Based Systems, Vol. 111, pp. 180192, Nov. 2016.

[22] C. Desrosiers and G. Karypis, "A Comprehensive Survey of Neighborhood-Based Recommendation Methods," in Recommender Systems Handbook, F. Ricci, L. Rokach, B. Shapira, and P.B. Kantor, Eds., New York, USA: Springer, 2011, pp. 107-144.

[23] T.D. Pessemier, S. Dooms, and L. Martens, "Comparison of Group Recommendation Algorithms," Multimedia Tools and Applications, Vol. 72, No. 3, pp. 2497-2541, Oct. 2014.

[24] S. Vargas and P. Castells, "Rank and Relevance in Novelty and Diversity Metrics for Recommender Systems," Proceedings of the 5th ACM Conference on Recommender Systems, 2011, pp. 109-116.

[25] D. Kotkov, J.A. Konstan, Q. Zhao, and J. Veijalainen, "Investigating Serendipity in Recommender Systems Based on Real User Feedback," Proceedings of the 33rd Annual ACM Symposium on Applied Computing, 2018, pp. 1341-1350.

[26] M. Kaminskas and D. Bridge, "Diversity, Serendipity, Novelty, and Coverage: A Survey and Empirical Analysis of Beyond-Accuracy Objectives in Recommender Systems," ACM Transactions on Interactive Intelligent Systems, Vol. 7, No. 1, pp. 1-42, Mar. 2017.

[27] J. Carbonell and J. Goldstein, "The Use of MMR, Diversity-Based Reranking for Reordering Documents and Producing Summaries,"
Proceedings of the 21st Annual International ACM SIGIR Conference on Research and Development in Information Retrieval, 1998, pp. 335-336.

[28] D. Kotkov, J. Veijalainen, and S. Wang, "How Does Serendipity Affect Diversity in Recommender Systems? A Serendipity-Oriented Greedy Algorithm," Computing, Vol. 102, No. 2, pp. 393-411, Feb. 2020.

[29] S. Sridharan, "Introducing Serendipity in Recommender Systems through Collaborative Methods," M.S. thesis, University of Rhode Island, Rhode Island, USA, 2014. [Online], https://digi talcommons.uri.edu/cgi/viewcontent.cgi?article=1455\&context=theses.

[30] P. Adamopoulos and A. Tuzhilin, "On Unexpectedness in Recommender Systems," ACM Transactions on Intelligent Systems and Technology, Vol. 5, No. 4, pp. 1-32, Jan. 2015.

[31] O. Chapelle, D. Metzler, Y. Zhang, and P. Grinspan, "Expected Reciprocal Rank for Graded Relevance," 18th ACM Conference on Information and Knowledge Management, 2009, pp. 621-630.

[32] B. Smyth and P. McClave, "Similarity vs. Diversity," International Conference on Case-Based Reasoning, 2001, pp. 347-361.

[33] C.N. Ziegler, S.M. McNee, J.A. Konstan, and G. Lausen, "Improving Recommendation Lists through Topic Diversification," Proceedings of the 14th international conference on World Wide Web, 2005, pp. 22-32.

[34] M.D. Ekstrand, F.M. Harper, M.C. Willemsen, and J.A. Konstan, "User Perception of Differences in Recommender Algorithms," Proceedings of the 8th ACM Conference on Recommender Systems, 2014, pp. 161-168.

[35] J.P. Kelly and D. Bridge, "Enhancing the Diversity of Conversational Collaborative Recommendations: A Comparison," Artificial Intelligence Review, Vol. 25, No. 1-2, pp. 79-95, Apr. 2006.

[36] M.T. Ribeiro, A. Lacerda, A. Veloso, and N. Ziviani, "Pareto-Efficient Hybridization for Multi-Objective Recommender Systems," Proceedings of the 6th ACM Conference on Recommender Systems, 2012, pp. 19-26.

[37] R.H.B. Christensen, "Ordinal: Regression Models for Ordinal Data," R Package Version 2015.6-28, access date: Nov. 25, 2018. [Online], http://cran.nexr.com/web/packages/ordinal/index.html. 